\title{
A Comparative Study of LQR and Integral Sliding Mode Control Strategies for Position Tracking Control of Robotic Manipulators
}

Preliminary Study

\author{
Nor Mohd Haziq Norsahperi \\ Universiti Putra Malaysia \\ Department of Electrical and Electronics Engineering, Faculty of Electrical Engineering \\ 43400, UPM Serdang, Selangor, Malaysia \\ nmhaziq@yahoo.com
}

\author{
Kumeresan A. Danapalasingam \\ Universiti Teknologi Malaysia \\ Centre for Artificial Intelligence and Robotics (CAIRO), School of Electrical Engineering, Faculty of Engineering \\ 81310, UTM Skudai, Johor, Malaysia \\ kumeresan@utm.my
}

\begin{abstract}
This paper provides a systematic comparative study of position tracking control of nonlinear robotic manipulators. The main contribution of this study is a comprehensive numerical simulation assessing position tracking performances and energy consumption of integral sliding mode control (ISMC), a linear-quadratic regulator with integral action (LQRT), and optimal integral sliding mode control (OISMC) under three conditions; namely, Case I) without the coupling effect, Case II) with the coupling effect on Link 1 only, and Case III) with the coupling effect on Link 2 only. The viability of the concept is evaluated based on three performance criteria, i.e., the step-response characteristics, position tracking error, and energy consumption of the aforementioned controllers. Based upon the simulation study, it has been found that OISMC offers performances almost similar to ISMC with more than 90\% improvement of tracking performance under several cases compared to LQRT; however, energy consumption is successfully reduced by $3.6 \%$ in comparison to ISMC. Energy consumption of OISMC can be further reduced by applying optimization algorithms in tuning the weighting matrices. This paper can be considered significant as a robotic system with high tracking accuracy and low energy consumption is highly demanded to be implemented in smart factories, especially for autonomous systems.
\end{abstract}

Keywords - sliding mode control, robotic manipulators, optimal control, Lyapunov method, linear-quadratic regulator.

\section{INTRODUCTION}

Industrial Revolution 4.0 (IR 4.0) promotes a "smart factory" concept in many sectors, especially in the manufacturing sector, and autonomous robots are an integral part of such concept. Autonomous robots are widely used in smart factories to enhance productivity and product quality $[1,2]$. Robotic manipulators are one of the operational robots that have been frequently installed in manufacturing plants due to their capability of performing various complex tasks, such as welding, painting, and assembling in any conditions [3]. However, robotic manipulators inherit nonlinear features, high coupling-effects, and parametric uncertainties [3-5]. Based on the aforementioned characteristics of robotic manipulators, designing prominent robust position tracking control is a challenging task for a control designer. Moreover, energy efficiency of engineering systems in industry has become a worldwide issue [6]. It is reported that $40 \%$ of total energy consumption in industries is consumed by electric motors [7]. As reported in [8], most robotic manipulators are indeed actuated by electric motors. Hence, energy consumption of robotic manipulators should be considered in designing a control system along with position tracking performance.

A considerable amount of literature has been published on position tracking control of robotic manipulators, such as proportional-integral-derivative (PID) control, as in $[9,10]$. Despite PID control prominent position tracking performances in robotic manipulator systems, a PID controller is only effective in a slow-speed system and very sensitive to external perturbation, as reported by $[11,12]$ and $[13,14]$, respectively. The PID controller exhibits a slow recovery speed due to the linear behaviour of PID control. The linear behaviour cannot deal with a nonlinear dynamic process of robotic manipulator systems [15], such as the Coriolis effect associated with the speed of robotic ma- 
nipulator systems. This issue may be explained by the fact that most nonlinear characteristics are ignored during the linear approximation process of robotic manipulator dynamics. In fact, the linear approximation process is a significant step in designing a classical controller, including PID control. Another prominent controller when it comes to dealing with nonlinear dynamics and robust tracking issues is intelligent control, as in $[16,17]$. Intelligent control offers an effective way to design a control system without a deep insight into the physical model of the system. However, the determination of its various parameters and complex rules is technically challenging and it relies on the designer's experience to construct an effective membership function $[5,18,19]$.

As many researchers have studied the position tracking problem, various modern controls have been developed to satisfy tracking performance, such as model predictive control (MPC) [20], computed torque control (CTC) [21], adaptive control $[22,23]$, and sliding mode control (SMC), as in $[3,24]$. In comparison to CTC, MPC offers low sensitivity against model imperfection. However, a major problem with MPC is that many parameters and coefficients should be properly selected, such as the prediction and control horizons, and also the penalisation factor to obtain satisfactory performance, as concluded by the authors in [25]. Nevertheless, adaptive control offers promising performance with a strong theoretical framework, as in [5], but it produces an aggressive control signal [26], which is particularly harmful to the actuator. Furthermore, the efficiency of adaptive control depends on the effectiveness of the learning scheme.

Among nonlinear control strategies, SMC is eminent as a simple and robust control strategy. SMC is of interest because it can keep the stability of a nonlinear system while maintaining the desired performance by addressing uncertainties in the system $[27,28]$. However, SMC is too expensive to be used for continuous operation as the control effort of a nonlinear controller scheme is enormous and aggressive, as mentioned in $[26,29]$. Moreover, SMC suffers from the robustness issue in the reaching phase. Thus far, several studies have been published on minimising control effort in robotic manipulator applications, such as in $[30,31]$. Both controllers in [30, 31] offer a promising result; however, such control schemes are complicated and sensitive to unmodelled dynamics [26, 32], especially for a high degree-of-freedom (DOF) system. As for the reaching phase issue of SMC, integral sliding mode control (ISMC) is introduced to eliminate the reaching phase [33], but ISMC still suffers from high control effort.

In view of these shortcomings, optimal integral sliding mode control (OISMC) is introduced in [34], where a linear-quadratic regulator (LQR) and ISMC are integrated to achieve multi-objective control conditions. By combining the LQR and ISMC, the excessive control effort of ISMC may be significantly reduced by properly selecting optimal parameters using the LQR approach. As a result, it inherits several merits, which are a robust control scheme against matched and unmatched uncertainties, and elimination of the SMC's reaching phase issue. However, the most important advantage of OISMC is that it can be manipulated to energy consumption of robotic manipulators by minimising its control effort. The performance of OISMC has been validated in similar applications by numerical simulation studies, as in [35] and [36]. However, previous studies in $[35,36]$ used OISMC to solve the robust tracking problem against matched and unmatched uncertainties without considering energy consumption. Collectively, these studies suggest that OISMC has the potential to reduce energy consumption by taking the control effort of OISMC in robotic manipulators as the key measurements in the cost function along with the position tracking error.

Therefore, the main contributions in this study are the linearisation of the 2-DOF nonlinear robotic manipulator dynamic model, comprehensive simulation studies of three control strategies, and comparative analyses of the three control strategies focusing on the position tracking performance and energy consumption. The three control strategies are as follows: the LQR controller with integral action (LQRT), conventional ISMC (ISMC), and OISMC. This paper is very significant because there is an absence of comprehensive robustness analysis with respect to the coupling effect and energy consumption. The rest of this paper is organised as follows. First, this paper introduces a nonlinear model of robotic manipulators and the model is transformed into the input and output forms. It is followed by the linearisation process of the nonlinear model. The second stage is concerned with the development of the three aforementioned control strategies. The last stage of this paper presents the findings and discussion of the research.

\section{ROBOTIC MANIPULATOR DYNAMIC MODEL}

The equation of the motion for an $n$-degree of freedom (DOF) robotic manipulators is derived by using the Lagrange-Euler (LE) technique, as in [37]. In the next subsection, both nonlinear and linear dynamic equations are presented based on 2-DOF robotic manipulators, as shown in Fig. 1.

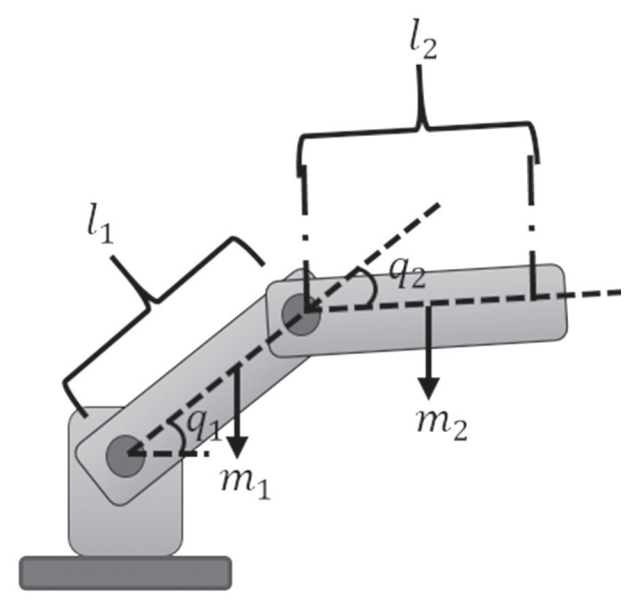

Fig. 1. Robotic manipulators 


\subsection{NONLINEAR DYNAMIC MODEL}

The dynamics of an n-DOF robotic manipulator can be expressed as:

$$
\tau=M(q) \ddot{q}+V(q, \dot{q})+G(q),
$$

where $M(q) \in R^{n \times n}$ is the inertia matrix, $\mathrm{V}(q, \dot{q}) \in R^{n}$ and $G(q) \in R^{n}$ denote the Coriolis/centripetal vector and the gravitational vector, respectively, $\tau \in R^{n}$ is the joint torque, and $q \in R^{n}$ denotes angular displacement vectors. (1) can be rearranged so that the angular acceleration vectors can be described as:

$$
\ddot{q}=M(q)^{-1}[\tau-V(q, \dot{q})-G(q)] .
$$

By defining the state vectors $x=[q, \dot{q}]^{\top}$ and $x=[\dot{q}, \ddot{q}]^{T}$, the nonlinear dynamic equation of robot manipulators can be rewritten as follows:

$$
\dot{x}=\left[\begin{array}{l}
\dot{x}_{1} \\
\dot{x}_{2}
\end{array}\right]=\left[\begin{array}{c}
\dot{q} \\
M(q)^{-1}[\tau-V(q, \dot{q})-G(q)]
\end{array}\right]
$$

Then, a nonlinear system without any uncertainties can be expressed in the canonical form of $\dot{x}=f(x, t)+b(x, t) u$ as:

$$
\dot{x}=\left[\begin{array}{l}
\dot{x}_{1} \\
\dot{x}_{2}
\end{array}\right]=\left[\begin{array}{c}
\dot{q} \\
M(q)^{-1}[-V(q, \dot{q})-G(q)]
\end{array}\right]+M(q)^{-1}\left[\begin{array}{l}
0 \\
I
\end{array}\right] u,
$$

where $u=\left\{\tau\right.$ "and" $\left.u \in R^{n}\right\}$ represents the vector control input and $I \in R^{n \times n}$ is the identity matrix. All vectors used in this paper are presented in Appendix I.

\subsection{LINEARISED DYNAMIC MODEL}

In this section, the linearisation of the nonlinear model is explained. To begin this process, some assumptions are made.

Assumption 2.1: The linear approximation of the nonlinear model is formulated by using the Taylor series expansion, where $\sin q \approx q$ and $\cos q \approx 1$ for a small deflection angle of the manipulators. $\dot{q}^{2}, q^{2}$, and $q \dot{q}$ are approximately small and neglected.

Assumption 2.2: Matrix $A, B$, and $C$ are controllable. Sufficient conditions for checking the controllability of the system imply that the controllable matrix is a nonsingular matrix and full rank, where:

$$
\begin{gathered}
\operatorname{rank}\left(M_{C}\right)=\operatorname{rank}\left(\left[\begin{array}{l}
B A B \\
\cdots
\end{array} A^{n-1} B\right]\right)=n \\
\operatorname{rank}\left(M_{o}\right)=\operatorname{rank}\left(\begin{array}{c}
C \\
C A \\
\vdots \\
C A^{n-1}
\end{array}\right)=n .
\end{gathered}
$$

From these assumptions, the state equation of robotic manipulators is presented in the continuous time linear system form as:

$$
\begin{array}{r}
\dot{x}(\mathrm{t})=A x(t)+B u(t) \\
y(t)=C x(t)+D u(t)
\end{array}
$$

where the state variables can be defined as in Appendix II, and for the linearised dynamic equation, the vectors can be presented as $x=\left[q_{1} q_{2} \dot{q}_{1} \dot{q}_{2}\right]^{\top}, \mathrm{y}=\left[q_{1} q_{2}\right]^{\top}$, and $\mathrm{u}=\left[\tau_{1} \tau_{2}\right]^{\top}$.

\section{CONTROLLER DESIGN}

In this section, three controllers are designed by using both derived dynamic equations in (4), (7), and (8). ISMC, the LQRT, and OISMC are designed to achieve high angular accuracy as the main objective. The tracking error vector, $e(t)$ can be defined as follows:

$$
e(t)=q_{r}(t)-q(t)
$$

where $q_{r} \in R^{n}$ denotes the desired angular position and $q \in R^{n}$ is the actual angular position of each joint.

\subsection{INTEGRAL SLIDING MODE CONTROL}

As described in the introduction, ISMC is well known as a robust control strategy. Moreover, it offers high precision for tracking problems and eliminates the reaching phase issue commonly associated with classical SMC [5]. There are two main steps in designing ISMC: 1 ) integral sliding function design $\left(s_{\text {ISMC }}\right)$, and 2 ) control law formulation $\left(u_{I S M C}\right)$. The control law, $u_{I S M C}$ is basically a combination between an equivalent control part, $u_{\text {eqISMC'}}$ and a switching control part, $u_{\text {SwISMC }}\left(u_{\text {ISMC }}\right.$ $\left.(t)=u_{\text {eqISMC }}(t)+u_{\text {SWISMC }}(t)\right)$. The integral sliding function $\left(s_{\text {ISMC }}\right)$ can be defined as:

$$
s_{I S M C}(t)=\dot{e}(t)+\alpha_{1} e(t)+\alpha_{2} \int_{0}^{t} e d t,
$$

where $\alpha_{1}$ and $\alpha_{2}$ are positive diagonal gain matrices. The time derivative of the sliding surface can be expressed as:

$$
\dot{s}_{I S M C}(t)=\ddot{e}(t)+\alpha_{1} \dot{e}(t)+\alpha_{2} e(t) .
$$

As $S_{\text {ISMC }}$ is successfully defined, the control law of ISMC $\left(u_{\text {ISMC }}\right)$ can be formulated. The ideal sliding conditions $\left(s_{I S M C}=\dot{s}_{I S M C}=0\right)$ should be conformed to keep the error trajectories on the sliding surface during the entire system response despite the presence of the matched uncertainty. The equivalent control, $u_{\text {eqISMC' }}$ can be obtained by solving the following:

$$
\dot{S}_{I S M C}(t)=\ddot{e}(t)+\alpha_{1} \dot{e}(t)+\alpha_{2} e(t)=0
$$

By taking $e^{\prime \prime}(t)=q_{r}-q "$ and (2) into (12), the equivalent control $\left(u_{\text {eqISMC }}\right)$ can be obtained as:

$$
\begin{gathered}
\ddot{q}_{r}-\ddot{q}+\alpha_{1} \dot{e}+\alpha_{2} e=0 \\
\ddot{q}_{r}-M(q)^{-1}[-V(q, \dot{q})-G(q)]-M(q)^{-1} u_{e q I S M C} \\
+\alpha_{1} \dot{e}+\alpha_{2} e=0,
\end{gathered}
$$


where $u_{\text {SwISMC }}(t)$ deals with lumped matched uncertainties, $\varepsilon$, such as parameter uncertainty, unmodelled dynamics, and external disturbance with a known upper bound.

$u_{e q I S M C}(t)=M(q)\left[\ddot{q}_{r}+\alpha_{1} \dot{e}+\alpha_{2} e\right]+V(q, \dot{q})+G(q)$

The control law of ISMC $\left(u_{I S M C}\right)$ can be rewritten by substituting (14) into $u_{I S M C}$ and this yields:

$$
u_{I S M C}(t)=u_{e q I S M C}(t)+u_{s w I S M C}(t),
$$

where $u_{\text {swISMC }}(t)=\delta \operatorname{sign}\left(s_{I S M C}\right)$ and $\delta$ is the gain matrix to improve the reachability of ISMC.

Proof: The existence of integral sliding motion can be proven by using the Lyapunov function candidate:

$$
\begin{gathered}
V_{I S M C}=\frac{1}{2} S_{I S M C}{ }^{T} S_{I S M C} \\
\dot{V}_{I S M C}=s_{I S M C^{T}} \dot{S}_{I S M C^{\prime}} \\
V_{I S M C}=s_{I S M C}{ }^{T}\left[\alpha_{1} \dot{e}+\alpha_{2} e+\ddot{q}_{r}-M(q)^{-1}[-V(q, \dot{q})-\right. \\
\left.G(q)]-M(q)^{-1} u_{I S M C}+M(q)^{-1} \varepsilon(t, x)\right]
\end{gathered}
$$

where $\varepsilon$ are lumped matched uncertainties. By substituting the control law in (15) into (17), it follows that:

$$
\begin{aligned}
\dot{V}_{I S M C}=s_{I S M C}{ }^{T} & {\left[\alpha_{1} \dot{e}+\alpha_{2} e+\ddot{q}_{r}-M(q)^{-1}[-V(q, \dot{q})-G(q)]\right.} \\
& -M(q)^{-1}\left[M(q) \mid \ddot{q}_{r}+\alpha_{1} \dot{e}+\alpha_{2} e\right] \\
& \left.\left.+V(q, \dot{q})+G(q)-u_{\text {SwISMC }}\right]\right] \\
\dot{V}_{I S M C} & =s_{I S M C}{ }^{T}\left[-\delta \operatorname{sign}\left(s_{I S M C}\right)+\varepsilon(t, x)\right] \\
& \leq\left\|s_{I S M C}\right\|[-\delta+\|\varepsilon(t, x)\|] .
\end{aligned}
$$

Thus, $V_{\text {ISMC }} \leq 0$ if $\delta \geq\|\varepsilon(t, x)\|$. It can be concluded that the error trajectories reach the sliding surface in finite time for all values of $\delta \geq\|\varepsilon(t, x)\|$. The proposed ISMC is illustrated in Fig. 2.

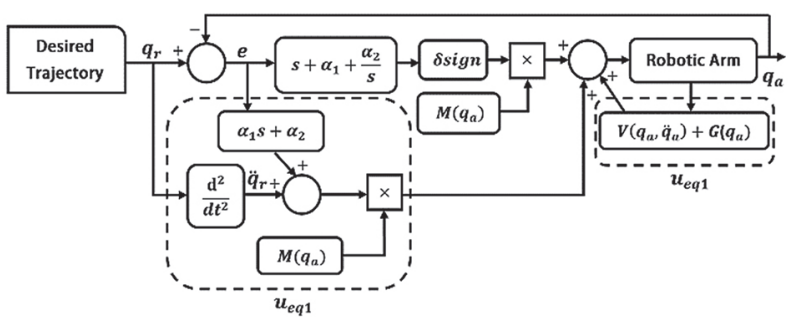

Fig. 2. Proposed ISMC architecture (s is a Laplace variable and q_a represents the robotic manipulator angular position)

\subsection{LINEAR-QUADRATIC REGULATOR WITH INTEGRAL ACTION}

LQR control has been attracting several researchers to reduce energy consumption of the system by considering energy consumption of the systems, such as in [38]. The common quadratic performance index can be defined as:

$$
J=\frac{1}{2} \int_{0}^{\infty}\left[\tilde{x}^{T}(t) Q_{L Q R T} \tilde{x}(t)+u_{C L Q R T}{ }^{T}(t) R_{L Q R T} u_{C L Q R T}(t)\right] d t
$$

where $u_{C L Q R T}$ is the control signal of the LQRT, while $Q_{L Q R T}$ and $R_{\text {LQRT }}$ are the symmetric positive definite state weighting matrices, respectively. However, as mentioned in [39], the conventional LQR controller cannot eliminate the steady-state error. Thus, the LQRT is introduced to improve tracking performance. In designing the LQRT, the linear dynamic equation as in (7) and (8) is used. By introducing the integral action into the system in (7), the augmented system can be formed as:

$$
\dot{\tilde{x}}(t)=\left[\begin{array}{c}
\dot{x} \\
\dot{x}_{e}
\end{array}\right]=\left[\begin{array}{cc}
A & 0 \\
-C & 0
\end{array}\right]\left[\begin{array}{c}
x \\
x_{e}
\end{array}\right]+\left[\begin{array}{c}
B \\
-D
\end{array}\right] u+\left[\begin{array}{l}
0 \\
I
\end{array}\right] r,
$$

where $\widetilde{A}=\left[\begin{array}{cc}A & 0 \\ -C & 0\end{array}\right]$ and $\widetilde{B}=\left[\begin{array}{c}B \\ -D\end{array}\right]$ matrices are used to solve the Riccati matrix equation for the LQRT to obtain a unique solution of the $P$ matrix. Moreover, $\dot{x}_{e}=-C x+r$, and $r$ defines the position reference. The Riccati matrix equation can be defined as:

$$
-P \tilde{A}-\tilde{A}^{T} P+P \tilde{B} R^{-1} \tilde{B}^{T} P-Q_{L Q R T}=0 .
$$

Finally, the control law of the LQRT can be defined as:

$$
u_{L Q R T}(t)=\left[K_{L Q R}-K_{e}\right] \widetilde{x}=-R^{-1} \widetilde{B}^{1} P \widetilde{x} .
$$

The LQRT algorithm can be mapped as in Fig. 3 .

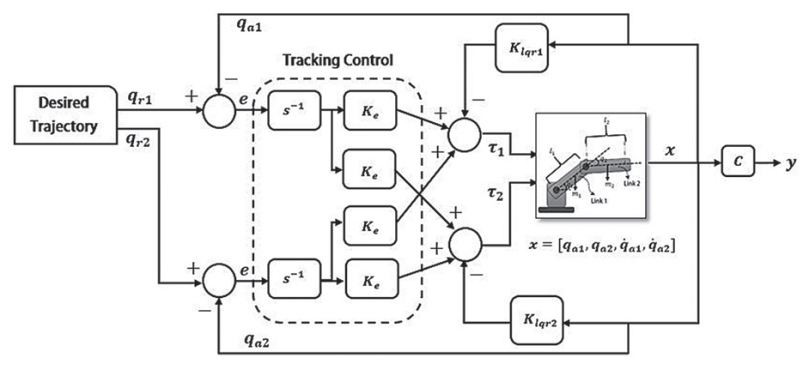

Fig. 3. Proposed LQRT architecture ( $\mathrm{s}$ is a Laplace variable and $q_{a}$ represents the robotic manipulator angular position)

\subsection{OPTIMAL INTEGRAL SLIDING MODE CONTROL}

As explained earlier, ISMC is a promising robust controller against matched uncertainty but it generates a high control signal. As mentioned by the authors in [26], a high control signal may increase energy consumption and degrade system performance. In view of these shortcomings, OISMC is proposed to reduce energy consumption and simultaneously maintain system performance. Another advantage of OISMC is that such control scheme can be easily designed for a multivariable 
system without amplifying unmatched uncertainty [40]. In this paper, the control law of OISMC is described as:

$$
u_{O I S M C}(t)=u_{o}(t)+u_{\text {SwOISMC }}(t)
$$

By combining the basic LQR control in $u_{-} 0$ and a switching control part $\left(u_{\text {swOISMC }}\right), u_{\text {OISMC }}$ can be rewritten as:

$$
u_{O I S M C}(t)=K_{\text {OISMC }} e(t)-\delta_{O} \operatorname{sign}\left(s_{O I S M C}\right),
$$

where $\delta_{O}$ is a scalar gain matrix to enforce the sliding motion, and $s_{O I S M C}$ is the integral sliding function of OISMC. $s_{\text {OISMC }}$ can be defined as:

$$
s_{\text {OISMC }}(t)=F e(t)-F e(0)-\int_{0}^{t} F\left(A e(t)+B u_{o}(t)\right)
$$

where $F=\left(B^{T} B\right)^{-1} B^{T}$ such that the matrix $F B$ becomes nonsingular and does not amplify the effect of unmatched uncertainty. A detailed explanation of the selection of the constant matrix $\mathrm{F}$ can be found in [40]. The cost function $J_{O}$ can be expressed as:

$$
J_{O}=\frac{1}{2} \int_{0}^{\infty}\left[e^{T}(t) Q_{O} e(t)+u_{C O}^{T}(t) R_{O} u_{C O}(t)\right] d t
$$

where $u_{c o}$ is the control signal of $u_{0}$ in OISMC, while $Q_{0}$ and $R_{O}$ are the symmetric positive definite state weighting matrices, respectively. $K_{\text {OISMC }}$ can be determined by using a similar approach to the classical LQR control:

$$
K_{O I S M C}=-R_{O}{ }^{-1} B^{T} P_{O}
$$

Also, matrix $P_{O}$ is the solution of the Riccati equation to minimise $J_{O}$ :

$$
-P_{O} A-A^{T} P_{O}+P_{O} B R_{O}^{-1} B^{T} P_{O}-Q_{O}=0 .
$$

Proof: The existence of integral sliding motion can be proven by using the Lyapunov function candidate as in [41]:

$$
V_{\text {OISMC }}=\frac{1}{2} S_{O I S M C}{ }^{T} S_{O I S M C}
$$

The time derivative of the Lyapunov function is defined as follows:

$$
\begin{gathered}
\dot{V}_{\text {OISMC }}=s_{\text {OISMC }}{ }^{T} \dot{s}_{\text {OISMC }} \\
\dot{V}_{\text {OISMC }}=s_{\text {OISMC }}\left[F\left(A e(t)+B u_{\text {OISMC }}(t)+\varepsilon(t, x)\right)\right. \\
\left.-F\left(A e(t)+B K_{\text {OISMC }} e(t)\right)\right] \\
\dot{V}_{\text {OISMC }}=s_{\text {OISMC }}{ }^{T} \\
F\left(A e(t)+B K_{\text {OISMC }} e(t)\right. \\
\left.\left.-B \delta_{O} \operatorname{sign}\left(s_{\text {OISMC }}\right)+\varepsilon(t, x)\right)-F\left(A e(t)+B K_{\text {OISMC }} e(t)\right)\right] \\
\dot{V}_{\text {OISMC }}=S_{\text {OISMC }}^{T}\left[-F \delta_{O} \operatorname{sign}\left(s_{\text {OISMC }}\right)+F \varepsilon(t, x)\right] \\
\leq\left\|s_{\text {OISMC }}\right\|[-F \delta+\|F \varepsilon(t, x)\|] .
\end{gathered}
$$

From (30), it can be concluded that integral sliding motion can be achieved for all values of $\delta \geq\|\varepsilon(t, x)\|$ and the system can be stabilized as long as $K_{\text {OISMC }}$ is computed by properly selecting weighting matrices $Q_{O}$ and $R_{O}$ [42]. The OISMC block diagram can be demonstrated as in Fig. 4.

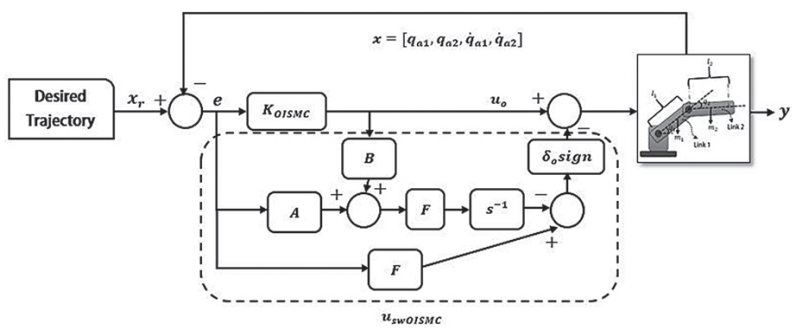

Fig. 4. Proposed OISMC Architecture (s is a Laplace variable and $q_{a}$ represents the robotic manipulator angular position)

\section{RESULTS AND DISCUSSION}

The previous section describes the controllers used in this study. The next part of this paper discusses the results obtained from the numerical simulation study. The designed controllers and a nonlinear model of 2-DOF robotic manipulators are mapped in MATLAB-Simulink for a simulation study. The main objective of the controller is to keep the angular position of each link at the desired trajectory under three separate cases and its energy consumption. The parameters are arbitrarily tuned until the satisfied tracking performance is achieved. Table 1 presents the selected parameters for ISMC.

Table 1. Parameters for ISMC

\begin{tabular}{cccccccc} 
& \multicolumn{3}{c}{ Link 1 } & \multicolumn{3}{c}{ Link 2 } \\
Controller & $\boldsymbol{a}_{1}$ & $\boldsymbol{a}_{2}$ & $\boldsymbol{\delta}$ & $\boldsymbol{a}_{1}$ & $\boldsymbol{a}_{2}$ & $\boldsymbol{\delta}$ \\
& 13 & 13 & 5 & 13 & 13 & 5
\end{tabular}

As for the LQRT and OISMC, the selected $Q_{\text {LQRT' }} R_{\text {LQRT }}$ $Q_{O^{\prime}} R_{O^{\prime}}$ and $\delta_{O}$ matrices for the LQRT and OISMC can be described as in (31) and (32), respectively:

$$
\begin{gathered}
Q_{L Q R T}=\left[\begin{array}{llllll}
1 & 0 & 0 & 0 & 0 & 0 \\
0 & 1 & 0 & 0 & 0 & 0 \\
0 & 0 & 1 & 0 & 0 & 0 \\
0 & 0 & 0 & 1 & 0 & 0 \\
0 & 0 & 0 & 0 & 1 & 0 \\
0 & 0 & 0 & 0 & 0 & 1
\end{array}\right]=I_{6 \times 6} \\
R_{L Q R T}=0.01 \times\left[\begin{array}{ll}
1 & 0 \\
0 & 1
\end{array}\right] \\
Q_{O}=\left[\begin{array}{llll}
1 & 0 & 0 & 0 \\
0 & 1 & 0 & 0 \\
0 & 0 & 1 & 0 \\
0 & 0 & 0 & 1
\end{array}\right]=I_{4 \times 4}, R_{O}=0.05 \times\left[\begin{array}{rr}
1 & 0 \\
0 & 1
\end{array}\right], \\
\delta_{O}=\left[\begin{array}{ll}
5 & 0 \\
0 & 5
\end{array}\right] .
\end{gathered}
$$


For the purpose of analysis, three case studies are conducted to evaluate tracking performances of the proposed controllers. The studies are described in the following cases:

Case I: Link 1 and Link 2 step responses without the coupling effect.

Case II: Link 1 step response with a dynamic input on Link 2.

Case III: Link 2 step response with a dynamic input on Link 1.

The desired trajectories for the step input and the dynamic input are defined as $0.7854 \mathrm{rad}$ and $0.7854 \sin (\mathrm{t})$, respectively. Moreover, the starting position for the dynamic input and the sampling period for all studies are set to $\pi / 2$ rad and $0.1 \mathrm{~ms}$, respectively. In order to assess the controller performance, three performance criteria are evaluated: 1 . Step response characteristics, 2. Mean square error (MSE), and 3. Energy of control input $(\mathrm{ECl}) . \mathrm{MSE}$ and $\mathrm{ECl}$ are measured by:

$$
\begin{gathered}
M S E=\frac{\sum_{J=1}^{N} e^{2}}{N} \\
E C I=\int_{0}^{t} u^{2} d u,
\end{gathered}
$$

where $N$ denotes the total number of sampling data.

\subsection{IDEAL STEP RESPONSE}

The first case aims to evaluate step response characteristics of the designed controllers and the results are shown in Fig. 5 and Table 2. By comparing the results in Fig. 5 and Table 2, it can be seen that in comparison to the LQRT, there is a significant reduction of the overshoot (os) and undershoot (us) in ISMC and OISMC step-input responses. ISMC and OISMC successfully reduce the overshoot (os) and undershoot approximately by 5\% and 31\%, respectively. Moreover, it is apparent from Table 2 that the time responses in terms of the rise time $(t)$ and settling time $\left(t_{s}\right)$ of ISMC and OISMC are very fast, i.e. almost $90 \%$ faster than the LQRT. These findings have important implications for developing robotic manipulators, especially for restricted space and cooperative-form applications, where fast response with low overshoot and undershoot are necessary features in the system performance.

Despite the improvement of some step-response characteristics by ISMC and OISMC, compared to the LQRT, there is a slight increase in the steady-state accuracy (e_ss) due to the chattering effect. This finding is unexpected because the LQRT is designed to optimise the control signal rather than the positioning precision. However, this value has no significant effect on the overall transient performance as the increment is small, i.e. approximately 0.1 to 0.4 milliradian. In addition, ISMC and OISMC still suffer from insubstantial magnitude of chattering, which may increase energy consumption.

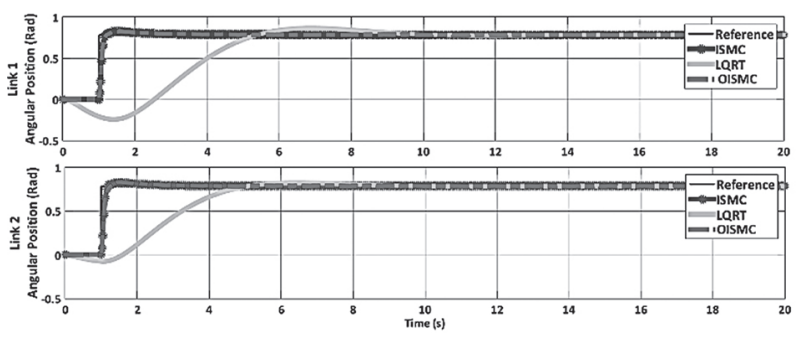

Fig. 5. Response of the designed controllers for Case I

Table 2. Step response characteristics of the designed controllers for Case I: a) Link 1 and (b) Link 2

\begin{tabular}{cccccc} 
Controller & $\boldsymbol{t}_{\boldsymbol{r}}(\boldsymbol{s})$ & $\boldsymbol{t}_{\boldsymbol{s}}(\boldsymbol{s})$ & os $(\%)$ & us $(\%)$ & $\boldsymbol{e}_{\text {ss }}(\mathbf{r a d})$ \\
\hline ISMC & 0.11 & 2.47 & 5.25 & 0 & 0.0001 \\
\hline OISMC & 0.12 & 2.59 & 5.52 & 0 & 0.0004 \\
LQRT & 2.13 & 9.17 & 10.06 & 31.22 & 0 \\
\hline
\end{tabular}

(a)

\begin{tabular}{cccccc} 
Controller & $\boldsymbol{t}_{\boldsymbol{r}}(\boldsymbol{s})$ & $\boldsymbol{t}_{\boldsymbol{s}}(\boldsymbol{s})$ & os $(\%)$ & us (\%) & $\boldsymbol{e}_{\text {ss }}(\mathbf{r a d})$ \\
\hline ISMC & 0.11 & 2.47 & 5.25 & 0 & 0.0001 \\
\hline OISMC & 0.13 & 2.6 & 6.18 & 0 & 0.0005 \\
\hline LQRT & 1.41 & 7.24 & 4.78 & 9.69 & 0 \\
\hline
\end{tabular}

(b)

\subsection{TRACKING PERFORMANCE}

The purpose of Case II and Case III studies is to measure tracking performances of the designed controllers under the high coupling effect of robotic manipulators, in which the opposite link is injected with the dynamic sinusoidal input. Tracking performances of the designed controllers are compared in Fig. 6 and Fig. 7. In Fig. 7, it can be seen that ISMC and OISMC give the lowest MSE values for all cases, whereas the LQRT shows a sluggish response. These results indicate that ISMC and OISMC offer a better position tracking control scheme to encounter the coupling effect for the robotic manipulator than the LQRT by approximately more than $90 \%$ reduction of MSE for all studies. Further analysis shows that the LQRT produces fragile tracking capability due to the high coupling effect. These results are likely to be related to the low control signal of the LQRT; consequently, the robotic manipulator does not receive a sufficient control signal to properly react from the unexpected perturbation. The next section discusses energy consumption of the designed controllers.
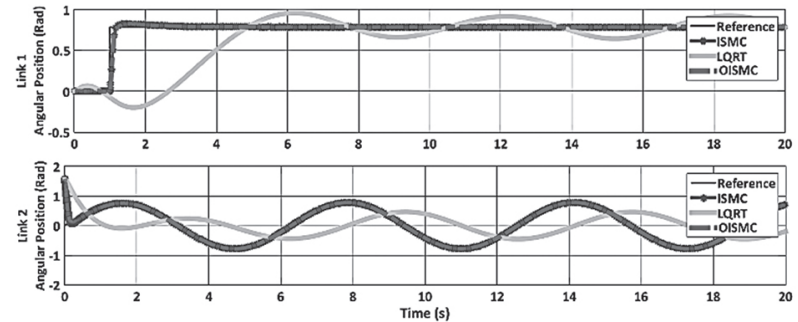

(a) 

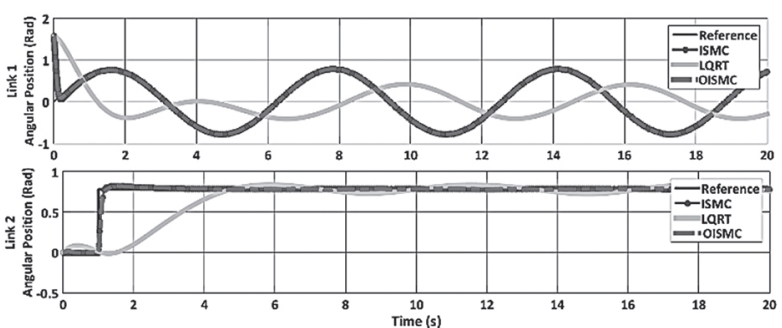

(b)

Fig. 6. Simulation results of the designed controllers: a) Case II and b) Case III

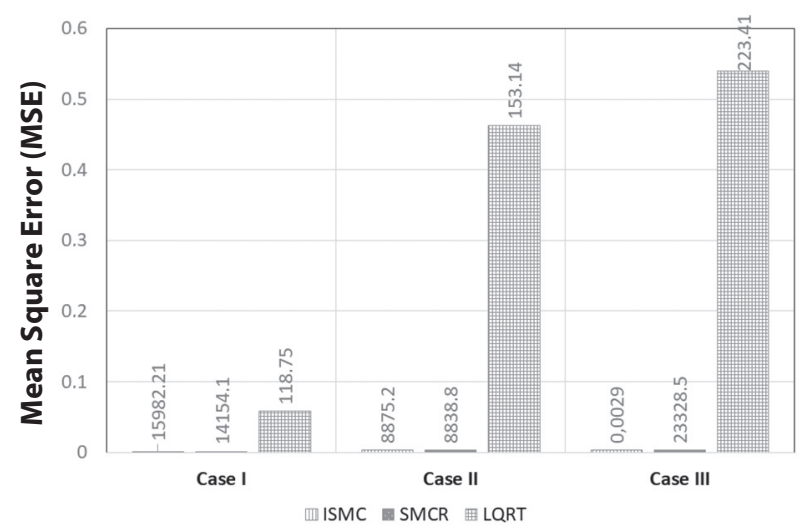

Fig. 7. MSEs of tracking errors

\subsection{ENERGY CONSUMPTION}

Energy consumption of each designed controller is compared in Fig. 8 and Fig. 9. In Fig. 9, the results provide the evidence that the LQRT offers low energy consumption compared to the ISMC and OISMC schemes, but it penalises position tracking performances of robotic manipulators. Perhaps the most important finding is that OISMC successfully reduces energy consumption by $3.6 \%$, while it offers almost similar performance compared to ISMC. This result may be explained by the fact that the designed regulator control can reduce the control signal computed by the equivalent control part $\left(u_{\text {eqISMC }}\right)$ in the ISMC scheme. It can be seen that the difference between the energy consumption of ISMC and OISMC may be small but the designed regulator control may be further improved as its $\mathrm{Q}$ and $\mathrm{R}$ matrices can be further tuned by using any optimisation technique. An implication of energy reduction is that OISMC can reduce the energy cost for continuous operation, and simultaneously, it may reduce the operational cost due to wear and tear on the actuator and mechanical structure.

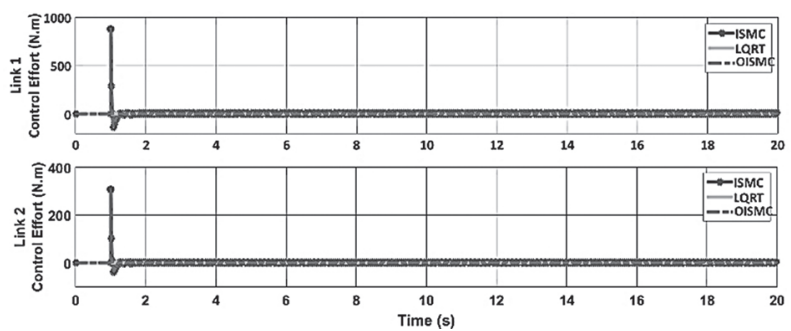

(a)
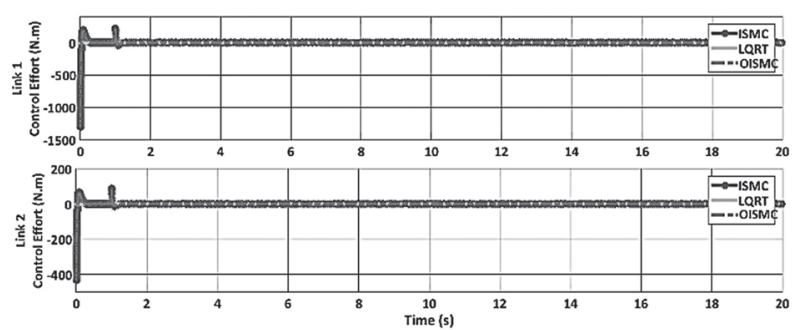

(b)

Fig. 8. Control inputs of joints: a) Case I, b) Case II, and c) Case III

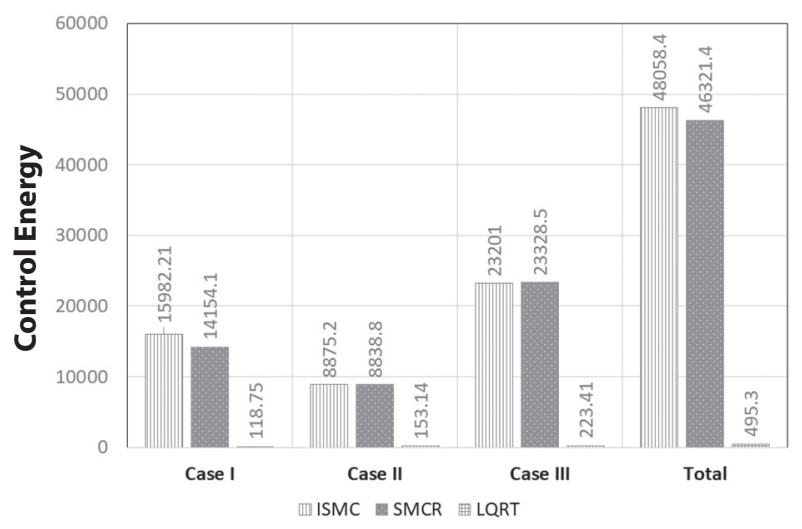

Fig. 9. ECls of control inputs

\section{CONCLUSION}

This study is set out to comprehensively compare the performances of ISMC, OISMC, and the LQRT. It can be concluded from that OISMC can replicate almost similar step input characteristics and tracking performance under various cases just like ISMC. The second major finding is the reduction of the control effort of OISMC, while maintaining such performance. Moreover, further analysis shows that, in comparison with ISMC and OISMC, the LQRT offers high steady-state accuracy and low energy consumption but it suffers from high sensitivity towards the coupling effect and a worse transient response. The current findings add to a growing literature on both the ISMC scheme and optimal control. As an extension to our work, it would be interesting to further optimise the weighting matrices in OISMC by using optimisation techniques. Further investigation and experimentation into OISMC are strongly recommended, especially on the robustness of OISMC under the presence of noise measurement and external torque disturbance.

\section{ACKNOWLEDGEMENT}

This work was funded by Universiti Teknologi Malaysia (UTM) through the internal grant, Research University Grant (GUP) UTMShine, Cost Center No. Q.J130000.2451.04G76. 


\section{REFERENCES:}

[1] M. Bahrin, F. Othman, N. Azli, M. Talib, "Industry 4.0: a review on industrial automation and robotic", Jurnal Teknologi, Vol. 78, No. 6-13, 2016, pp. 137-143.

[2] B. Bayram, G. Ince, "Advances in robotics in the era of industry 4.0: managing the digital transformation", Springer, Cham, 2018, pp. 187-200.

[3] S.A. Ajwad, J. Iqbal, R.U. Islam, A. Alsheikhy, A. Almeshal, A. Mehmood, “Optimal and robust control of multi dof robotic manipulator: design and hardware realization", Cybernetics and Systems, Vol. 49, No. 1, 2018, pp. 77-93.

[4] G. Herrmann, J. Na, M.N. Mahyuddin, “Novel Robust Adaptive Algorithms for Estimation and Control: Theory and Practical Examples", Elsevier, 2016.

[5] J. Lee, P.H. Chang, M. Jin, “Adaptive integral sliding mode control with time-delay estimation for robot manipulators", IEEE Transactions on Industrial Electronics, Vol. 64, No. 8, 2017, pp. 6796-6804.

[6] G. May, I. Barletta, B. Stahl, M. Taisch, “Energy management in production: a novel method to develop key performance indicators for improving energy efficiency", Applied Energy, Vol. 149, No. 2015, 2015, pp. 46-61.

[7] R. Saidur, "A review on electrical motors energy use and energy savings", Renewable and Sustainable Energy Reviews, Vol. 14, No. 3, 2010, pp. 877-898.

[8] J. Iqbal, R.U. Islam, S.Z. Abbas, A.A. Khan, S.A. Ajwad, "Automating industrial tasks through mechatronic systems - a review of robotics in industrial perspective", Tehnicki vjesnik - Technical Gazette, Vol. 23, No. 3, 2016, pp. 917-924.

[9] Y. Su, C. Zheng, "PID control for global finite-time regulation of robotic manipulators", International Journal of Systems Science, Vol. 48, No. 3, 2016, pp. 547-558.

[10] H. Anwaar, Y. Yixin, S. ljaz, M.A. Ashraf, W. Anwaar, "Fractional order based computed torque control of 2-link robotic arm", Advances in Science and Technology Research Journal, Vol. 12, No. 1, 2018, pp. 273-284.

[11] W. Shang, S. Cong, "Robust nonlinear control of a planar 2-dof parallel manipulator with redundant actuation", Robotics and Computer-Integrated Manufacturing, Vol. 30, No. 6, 2014, pp. 597-604.

[12] B. Zhao, B. Xian, Y. Zhang, X. Zhang, "Nonlinear robust adaptive tracking control of a quadrotor uav via immersion and invariance methodology", IEEE Transactions on Industrial Electronics, Vol. 62, No. 5, 2015, pp. 2891-2902.

[13] J. Jung, V.Q. Leu, T.D. Do, E. Kim, H.H. Choi, "Adaptive pid speed control design for permanent magnet synchronous motor drives", IEEE Transactions on Power Electronics, Vol. 30, No. 2, 2015, pp. 900-908.

[14] N.M.H. Norsahperi, S. Ahmad, S.F. Toha, I.A. Mahmood, N.H.H.M. Hanif, "Robustness analysis of fractional order pid for an electrical aerial platform †", Journal of Mechanical Science and Technology, Vol. 32, No. 11, 2018, pp. 5411-5419.

[15] S.A. Ajwad, J. lqbal, M.I. Ullah, A. Mehmood, "A systematic review of current and emergent manipulator control approaches", Frontiers of Mechanical Engineering, Vol. 10, No. 2, 2015, pp. 198-210.

[16] M.J. Er, Y. Gao, "Robust adaptive control of robot manipulators using generalized fuzzy neural networks", IEEE Trans. Ind. Electron., Vol. 50, No. 3, 2003, pp. 620-628

[17] A. Kumar, V. Kumar, "Evolving an interval type-2 fuzzy pid controller for the redundant robotic manipulator", Expert Systems with Applications, Vol. 73, 2017, pp. 161-177.

[18] R.J. Lian, "Adaptive self-organizing fuzzy slidingmode radial basis-function neural-network controller for robotic systems", IEEE Transactions on Industrial Electronics, Vol. 61, No. 3, 2014, pp. 1493-1503.

[19] N.M.H. Norsahperi, K.A. Danapalasingam, “Neurocontrol Design for an Aerodynamics System: Simple Backpropagation Approach", Lecture Notes in Electrical Engineering, Springer, 2019, pp. 3-9.

[20] T. Faulwasser, T. Weber, P. Zometa, R. Findeisen, "Implementation of nonlinear model predictive path-following control for an industrial robot", IEEE Transactions on Control Systems Technology, Vol. 25, No. 4, 2017, pp. 1505-1511.

[21] A. Zubizarreta, M. Marcos, I. Cabanes, C. Pinto, “Design and implementation of sliding mode algo- 
rithm: applied to robot manipulator-a review", International Journal of Robotics and Automation, Vol. 2, No. 5, 2011, pp. 265-282.

[22] S. Mondal, C. Mahanta, "Adaptive second order terminal sliding mode controller for robotic manipulators", Journal of the Franklin Institute, Vol. 351, No. 4, 2014, pp. 2356-2377.

[23] J. Baek, M. Jin, S. Han, "A new adaptive slidingmode control scheme for application to robot manipulators", IEEE Transactions on Industrial Electronics, Vol. 63, No. 6, 2016, pp. 3628-3637.

[24] L. Zhang, L. Liu, Z. Wang, Y. Xia, “Continuous finitetime control for uncertain robot manipulators with integral sliding mode", IET Control Theory \& Applications, Vol. 12, No. 11, 2018, pp. 1621-1627.

[25] S.A. Ajwad, M.I. Ullah, K. Baizid, J. Iqbal, “A comprehensive state-of-the-art on control of industrial articulated robots", Journal of the Balkan Tribological Association, Vol. 20, No. 4, 2014, pp. 499-521.

[26] J. Iqbal, M. Ullah, S.G. Khan, B. Khelifa, S. Ćuković, "Nonlinear control systems-a brief overview of historical and recent advances", Nonlinear Engineering, Vol. 6, No. 4, 2017, pp. 301-312.

[27] O. Barambones, P. Alkorta, "Position control of the induction motor using an adaptive sliding-mode controller and observers", IEEE Transactions on Industrial Electronics, Vol. 61, No. 12, 2014, pp. 6556-6565.

[28] J.E. Slotine, S.S. Sastry, "Tracking control of nonlinear systems using sliding surfaces with application to robot manipulators", International Journal of Control, Vol. 38, No. 2, 1983, pp. 1-56.

[29] W.B. Lin, H.K. Chiang, "Super-twisting algorithm second-order sliding mode control for a synchronous reluctance motor speed drive", Mathematical Problems in Engineering, 2013, pp. 1-9.

[30] S.G. Khan, G. Herrmann, T. Pipe, C. Melhuish, A. Spiers, "Safe adaptive compliance control of a humanoid robotic arm with anti-windup compensation and posture control", International Journal of Social Robotics, Vol. 2, No. 3, 2010, pp. 305-319.

[31] H. Tian, "A repetitive learning method based on sliding mode for robot control with actuator saturation", Journal of Dynamic Systems, Measurement, and Control, Vol. 137, No. 6, 2015, pp. 064505.
[32] M.C. Turner, "Positive $\mu$ modification as an antiwindup mechanism", Systems and Control Letters, Vol. 102, 2017, pp. 15-21.

[33] S.U. Din, Q. Khan, F.-U. Rehman, R. Akmeliawanti, "A comparative experimental study of robust sliding mode control strategies for underactuated systems", IEEE Access, Vol. 5, 2018, pp. 1927-1939.

[34] V. Utkin, J. Guldner, J. Shi, "Sliding Mode Control in Electromechanical Systems", 2nd ed., Taylor \& Francis Group, 2009.

[35] M.N. Ahmad, H.S.O. Johari, "Application of Proportional-Integral Sliding Mode Tracking Controller to Robot Manipulators", Proceedings of 2003 IEEE Conference on Control Applications, IEEE, 2003, pp. 87-92.

[36] M. Jouini, S. Dhahri, A. Sellami, "Combination of integral sliding mode control design with optimal feedback control for nonlinear uncertain systems", Transactions of the Institute of Measurement and Control, Vol. 41, No. 5, 2019, pp. 1331-1339.

[37] S.B. Niku, "Introduction to Robotics", 2nd ed., John Wiley \& Son, 2010.

[38] S. Ghosh, S. Kamalasadan, "An energy functionbased optimal control strategy for output stabilization of integrated dfig-flywheel energy storage system", IEEE Transactions on Smart Grid, Vol. 8, No. 4, 2017, pp. 1922-1931.

[39] B. Kedjar, K. Al-Haddad, “DSP-based implementation of an lqr with integral action for a three-phase three-wire shunt active power filter", IEEE Transactions on Industrial Electronics, Vol. 56, No. 8, 2009, pp. $2821-2828$.

[40] F. Castaños, L. Fridman, "Analysis and design of integral sliding manifolds for systems with unmatched perturbations", IEEE Transactions on Automatic Control, Vol. 51, No. 5, 2006, pp. 853-858.

[41] M.T. Hamayun, E. Christopher, H. Alwi, “Integral Sliding Mode Control Variable", Studies in Systems, Decision and Control, 61st ed., Springer, 2016, pp. 17-37.

[42] N. Arab, B. Kedjar, A. Javadi, K. Al-Haddad, "A multifunctional single phase grid integrated residential solar pv systems based on lqr control", IEEE Transactions on Industry Applications, Vol. 55, No. 2, 2018, pp. 2099-2109. 


\section{APPENDIX I}

A nonlinear model of 2-DOF robotic manipulators:

$M(q)=\left[\begin{array}{ll}M_{11} & M_{12} \\ M_{21} & M_{22}\end{array}\right], V(q, \dot{q})=\left[\begin{array}{l}V_{11} \\ V_{21}\end{array}\right], G(q)=\left[\begin{array}{l}G_{11} \\ G_{21}\end{array}\right]$

$M_{11}=\frac{1}{3} m_{1} l_{1}{ }^{2}+m_{2} l_{1}{ }^{2}+\frac{1}{3} m_{2} l_{2}{ }^{2}+m_{2} l_{1} l_{2} \cos q_{2}$

$M_{12}=M_{21}=\frac{1}{3} m_{2} l_{2}{ }^{2}+\frac{1}{2} m_{2} l_{1} l_{2} \cos q_{2}$

$M_{22}=\frac{1}{3} m_{2} l_{2}^{2}$

$V_{11}=-m_{2} l_{1} l_{2}\left(\sin q_{2}\right) \dot{q}_{1} \dot{q}_{2}-\frac{1}{2} m_{2} l_{1} l_{2}\left(\sin q_{2}\right) \dot{q}_{2}^{2}$

$V_{21}=\frac{1}{2} m_{2} l_{1} l_{2}\left(\sin q_{2}\right) \dot{q}_{1}^{2}$

$G_{11}=\frac{1}{2} m_{1} g l_{1}\left(\cos q_{1}\right)+m_{2} g l_{1}\left(\cos q_{1}\right)$

$$
+\frac{1}{2} m_{2} g l_{2}\left(\cos \left(q_{1}+q_{2}\right)\right)
$$

$G_{21}=\frac{1}{2} m_{2} g l_{2}\left(\cos \left(q_{1}+q_{2}\right)\right)$,

where, $q, \dot{q}$, and $\ddot{q}$ denote the angular displacement, angular velocity, and angular acceleration of Link 1 and Link 2, respectively. $I_{1}$ and $I_{2}$ are the lengths of Link 1 and Link 2, respectively. The parameters are defined as in Table 3.

Table 3. Robotic manipulator parameters

\begin{tabular}{|ccc} 
Parameters (Units) & Symbols & Values \\
\hline Mass of Link 1 $(\mathrm{kg})$ & $\mathrm{m}^{1}$ & 1 \\
\hline Mass of Link 2 $(\mathrm{kg})$ & $\mathrm{m}^{2}$ & 2 \\
\hline Length of Link $(\mathrm{m})$ & $P^{2}=l^{1}=l$ & 0.5 \\
\hline Gravitational acceleration $\left(\mathrm{m} / \mathrm{s}^{2}\right)$ & $g$ & 9.81
\end{tabular}

\section{APPENDIX II}

A linearised model of 2-DOF robotic manipulators:

$$
\begin{gathered}
M(q)^{-1}=\frac{1}{M_{11} M_{22}-M_{12} M_{21}}\left[\begin{array}{cc}
M_{22} & -M_{12} \\
-M_{21} & M_{11}
\end{array}\right] \\
=\left[\begin{array}{ll}
I M_{11} & I M_{12} \\
I M_{21} & I M_{22}
\end{array}\right]
\end{gathered}
$$

A linearised model of robotic manipulators based on Assumption 2.1:

$$
\begin{aligned}
& M_{11}=\frac{1}{3} m_{1} l^{2}+\frac{4}{3} m_{2} l^{2}+m_{2} l^{2}=\frac{1}{3} m_{1} l^{2}+\frac{7}{3} m_{2} l^{2} \\
& M_{12}=M_{21}=\frac{1}{3} m_{2} l^{2}+\frac{1}{2} m_{2} l^{2}=\frac{5}{6} m_{2} l^{2} \\
& M_{22}=\frac{1}{3} m_{2} l^{2} \\
& V_{11}=m_{2} l^{2} q_{2} \dot{q}_{1} \dot{q}_{2}+\frac{1}{2} m_{2} l^{2} q_{2} \\
& V_{21}=-\frac{1}{2} m_{2} l^{2} q_{2} \\
& G_{11}=-\frac{1}{2} m_{1} g l-\frac{3}{2} m_{2} g l \\
& G_{21}=-\frac{1}{2} m_{2} g l \\
& \dot{x}_{1}=f_{1}=\dot{q}_{1} \\
& \dot{x}_{2}=f_{2}=\dot{q}_{2} \\
& \dot{x}_{3}=f_{3}=I M_{11}\left[-V_{11}-G_{11}+\tau_{1}\right] \\
& +I M_{12}\left[-V_{21}-G_{21}+\tau_{2}\right] \\
& \dot{x}_{4}=f_{4}=I M_{21}\left[-V_{11}-G_{11}+\tau_{1}\right] \\
& +I M_{22}\left[V-21-G_{21}+\tau_{2}\right] \\
& A=\left[\begin{array}{cccc}
0 & 0 & 1 & 0 \\
0 & 0 & 0 & 1 \\
\frac{\partial f_{3}}{\partial x_{1}} & \frac{\partial f_{3}}{\partial x_{2}} & \frac{\partial f_{3}}{\partial x_{3}} & \frac{\partial f_{3}}{\partial x_{4}} \\
\frac{\partial f_{4}}{\partial x_{1}} & \frac{\partial f_{4}}{\partial x_{2}} & \frac{\partial f_{4}}{\partial x_{3}} & \frac{\partial f_{4}}{\partial x_{4}}
\end{array}\right] \\
& B=\left[\begin{array}{cc}
0 & 0 \\
0 & 0 \\
I M_{11} & I M_{12} \\
I M_{21} & I M_{22}
\end{array}\right] \text { and } C=\left[\begin{array}{llll}
1 & 0 & 0 & 0 \\
0 & 1 & 0 & 0
\end{array}\right]
\end{aligned}
$$

where $\mathrm{x}=\left[\begin{array}{llll}q_{1} & q_{2} & \dot{q}_{1} & \dot{q}_{2}\end{array}\right]^{\top}$. 


\section{APPENDIX III}

Raw data of tracking performances:

Case I:

\begin{tabular}{|ccc} 
Controller & MSE1 & MSE2 \\
\hline ISMC & $5.84 \times \mathrm{e}^{-4}$ & $5.84 \times \mathrm{e}^{-4}$ \\
\hline OISMC & $5.95 \times \mathrm{e}^{-4}$ & $5.95 \times \mathrm{e}^{-4}$ \\
\hline LQRT & 0.0385 & 0.0193 \\
\hline
\end{tabular}

Case II:

\begin{tabular}{ccc} 
Controller & MSE1 & MSE2 \\
\hline ISMC & $5.92 \times \mathrm{e}^{-4}$ & 0.0023 \\
\hline OISMC & $5.95 \times \mathrm{e}^{-4}$ & 0.0023 \\
\hline LQRT & 0.0461 & 0.4163 \\
\hline
\end{tabular}

Case III:

\begin{tabular}{ccc} 
Controller & MSE1 & MSE2 \\
\hline ISMC & 0.0023 & $5.9 \times \mathrm{e}^{-4}$ \\
\hline OISMC & 0.0023 & $6.17 \times \mathrm{e}^{-4}$ \\
\hline LQRT & 0.5194 & 0.0207
\end{tabular}

where MSE1 and MSE2 are the MSE of Link 1 and Link 2 , respectively.

\section{APPENDIX IV}

Raw data of energy consumption:

Case I:

\begin{tabular}{|cccc|}
\hline Controller & Link 1 & Link 2 & Total \\
\hline ISMC & 14257 & 1725.20 & 15982.2 \\
\hline OISMC & 12659 & 1495.1 & 14154.1 \\
\hline LQRT & 104.05 & 14.7 & 118.75 \\
\hline
\end{tabular}

Case II:

\begin{tabular}{|cccc|} 
Controller & Link 1 & Link 2 & Total \\
\hline ISMC & 7785.5 & 1089.7 & 8875.2 \\
\hline OISMC & 7751.3 & 1087.5 & 8838.8 \\
\hline LQRT & 127.54 & 25.60 & 153.14 \\
\hline
\end{tabular}

Case III:

\begin{tabular}{|cccc|} 
Controller & Link 1 & Link 2 & Total \\
\hline ISMC & 20855 & 2346 & 23201 \\
\hline OISMC & 20963 & 2365.5 & 23328.5 \\
\hline LQRT & 196.39 & 27.02 & 223.41 \\
\hline
\end{tabular}

